\title{
Robust exponential stabilization for a class of nonlinear uncertain systems with time-varying delays
}

\author{
MENG LIU, YALI DONG*，XINYUE TANG \\ School of Mathematical Sciences \\ Tiangong University \\ Tianjin 300387 \\ CHINA
}

\begin{abstract}
This paper is concerned with the problem of robust exponential stabilization for a class of nonlinear uncertain systems with time-varying delays. By using appropriately chosen Lyapunov-Krasovskii functional, together with the Finsler's lemma, sufficient conditions for exponential stability of nonlinear uncertain systems with time-varying delays are proposed in terms of linear matrix inequality (LMI). Then, novel sufficient conditions are developed to ensure the nonlinear uncertain system with time-varying delay is robust exponentially stabilizable in terms of linear matrix inequality with state feedback control. Finally, a numerical example is given to illustrate the efficiency of proposed methods.
\end{abstract}

Key-Words: - Exponential stabilization; nonlinear uncertain systems; time-varying delay; state feedback controller linear matrix inequality (LMI)

Received: April 25, 2021. Revised: September 26, 2021. Accepted: November 23, 2021. Published: December 20, 2021.

\section{Introduction}

During the past twenty years, nonlinear system have been extensively studied and successfully applied to various science and engineering fields such as mechanics, electrical engineering, automatic control and so on. Stability analysis and control design of nonlinear systems are very important research topics, which have attracted extensive attention from researchers [1-6]. In [1], stabilization of nonlinear time-delay systems was considered. $\mathrm{He}$ et al. [3] studied the global sampled-data output feedback stabilization for a class of stochastic nonlinear systems with time-varying delay. In [5], Dong et al. investigated exponential stabilization and $L_{2}$-gain for uncertain switched nonlinear systems with interval time-varying delay. Recently, Dong et al. [7] investigated robust exponential stabilization for uncertain neutral neural networks with time-varying delays by periodically intermittent control.

Time delays, both constant and time varying, are frequently encountered in various engineering, biological, chemical systems and economic systems. The existence of time delay worsens the dynamic performance of a system and even leads to instability of the system. Therefore, time-delay systems have been studied by many researchers [8-10]. Stability analysis and stabilizing controller design are key issues in the study of time-delay systems [11-13]. In [12], Hou et al. considered the robust exponential stability for discrete-time switched Hopfield neural networks with time delay. Zhang et al. [13] dealt with observer-based output feedback controller for discrete-time fuzzy systems.

On the other hand, the problem of robust stabilization of systems with uncertainties has also received much attention in recent years [14-16]. Delay-dependent exponential stabilization for uncertain linear systems with interval nondifferentiable time-varying delays was studied in [14]. In [15], Dong et al. considered the exponential stability and stabilization for uncertain discrete-time periodic systems with time-varying delay. 
Motivated by the above discussion, this paper investigates the stability and stabilization for a class of nonlinear uncertain systems with time-varying delays. By using appropriately chosen LyapunovKrasovskii functional, together with the Finsler's lemma, new sufficient conditions for exponential stability in terms of linear matrix inequalities are proposed for nonlinear uncertain time delay system. Then, we give a novel criterion which guarantees that the nonlinear uncertain system with time-varying delay is exponentially stabilizable via state feedback control. Finally, a numerical example is given to illustrate the less conservatism and effectiveness.

The paper is organized as follows. Section 2 states the problem formulation and preliminaries. Section 3 presents the main results for stability analysis and controller design by using Lyapunov-Krasovskii functional method. We present sufficient conditions for the exponential stabilization of the nonlinear ncertain system with time-varying delay. A numerical example is given in Section 4. Finally, the conclusion is given in Section 5.

Notations: $A^{T}$ denotes the matrix transpose of $A$. A symmetric positive (negative) definite matrix is expressed by $A>0(A<0) . \quad R^{n}$ and $R^{n \times m}$ stand for Euclidean $n$-space and the set of all $n \times m$ real matrices, respectively. $\lambda_{\max }(A)$ and $\lambda_{\min }(A)$ be the maximum and minimum eigenvalues for a given matrix $A$. The symbol " * " is used to indicate the elements induced by symmetry; The notation $\|\cdot\|$ denotes the Euclidean vector norm.

\section{System formulation preliminaries}

Consider the following system with time-varying dela

$$
\left\{\begin{aligned}
\dot{x}(t)= & {[A+\Delta A] x(t)+[B+\Delta B] x(t-\tau(t)) } \\
& +H u(t)+f(x(t)) \\
x(t)= & \phi(t), \quad t \in\left[-\tau_{2}, 0\right] .
\end{aligned}\right.
$$

where $x(t) \in R^{n}$ is the state vector, $u(t) \in R^{m}$ is the control input. $A, B, H$, are known real constant matrices of appropriate dimensions. The functio $\tau(t)$ is the time-varying delay satisfying

$$
0<\tau_{1} \leq \tau(t) \leq \tau_{2}, \quad \dot{\tau}(t) \leq \mu<1,
$$

where $\tau_{1}, \tau_{2}, \mu$ are positive constants. $\phi(t) \in L_{2}\left[-\tau_{2}, 0\right]$ is the initial function. The function $f(x(t))$ is a known nonlinear function satisfying $f(0)=0$ and the Lipschitz condition:

$$
\left\|f\left(x_{1}\right)-f\left(x_{2}\right)\right\| \leq \rho\left\|x_{1}-x_{2}\right\|, \forall x_{1}, x_{2} \in R^{n},
$$

where $\rho$ is the Lipschitz constant. $\Delta A(t)$ and $\Delta B(t)$ are time-varying matrices satisfying:

$$
[\Delta A(t) \quad \Delta B(t)]=M S(t)\left[\begin{array}{ll}
H_{1} & H_{2}
\end{array}\right],
$$

where $M, H_{1}, H_{2}$ are known matrices and $S(t)$ is an unknown time-varying matrix satisfying the following condition:

$$
S^{T}(t) S(t) \leq I, \quad \forall t>0 .
$$

Definition 1. [17] The system (1) with $u(t)=0$ is said to be exponentially stable with decay rate $\kappa$, if there exist scalars $\kappa>0$ and $\gamma>0$ satisfying

$$
\|x(t)\| \leq \gamma\|\phi\| e^{-k t}, \quad t \geq 0,
$$

where $\|\phi\|=\sup _{-\tau_{2}<\theta<0}\|\phi(\theta)\|$.

Lemma 1. (Finsler's lemma [18]) Consider $x(t) \in R^{n}, \quad \Phi \in R^{n \times n}$ and $U \in R^{m \times n}$ such that $\operatorname{rank}(U)=r<n$. Then, the following conditions are equivalent:

1. $x^{T} \Phi x<0, \forall x \in R^{n} \quad$ such that $x \neq 0$ and $U x=0$.

2. $\exists F \in R^{n \times m}$ satisfying $\Phi+F U+U^{T} F^{T}<0$.

Lemma 2. [10] For any time-varying matrix $S(t)$ satisfying $S^{T}(t) S(t) \leq I$ and a scalar $\eta$, the following condition is satisfied:

$$
M S(t) N+N^{T} S^{T}(t) M^{T} \leq \eta^{-1} M M^{T}+\eta N^{T} N,
$$

where $M, N$ are known matrices with appropriate dimensions.

\section{Main Results}

Consider system (1) with $u(t)=0$ and let $\bar{A}=A+\Delta A(t), \bar{B}=B+\Delta B(t)$. We have 


$$
\left\{\begin{array}{l}
\dot{x}(t)=\bar{A} x(t)+\bar{B} x(t-\tau(t))+f(x(t)), \\
x(t)=\phi(t), \quad t \in\left[-\tau_{2}, 0\right] .
\end{array}\right.
$$

The following theorem presents sufficient conditions to guarantee that the time-delay system (7) is exponentially stable.

Theorem 1. For given parameters $\varepsilon_{1}, \varepsilon_{2}, \varepsilon_{3}, \varepsilon_{4}$, $\rho>0, \alpha<0,0<\tau_{1} \leq \tau_{2}$, and $\mu<1$, the system (7) is exponentially stable with decay rate $\kappa=-\frac{\alpha}{2}$, if there exist symmetric positive definite matrices $P$, $\bar{Q}, Q, R, X$ and a positive scalar $\eta$, such that the following LMI holds:

$$
\Pi=\left[\begin{array}{cc}
\Psi_{11} & \Psi_{12} \\
* & \Psi_{22}
\end{array}\right]<0,
$$

where

$$
\begin{aligned}
& \Psi_{11}=\left[\begin{array}{cccc}
\Pi_{11} & \Pi_{12} & \varepsilon_{3} A^{T} X^{T} & \Pi_{14} \\
* & \Pi_{22} & \varepsilon_{3} X^{T} & \Pi_{24} \\
* & * & -e^{\alpha \tau_{1}} Q & \varepsilon_{3} X B \\
* & * & * & \Pi_{44}
\end{array}\right], \\
& \Psi_{12}=\left[\begin{array}{ccccc}
0 & \varepsilon_{1} X & \varepsilon_{1} X M & \eta H_{1}^{T} & I \\
0 & \varepsilon_{2} X & \varepsilon_{2} X M & 0 & 0 \\
0 & \varepsilon_{3} X & \varepsilon_{3} X M & 0 & 0 \\
0 & \varepsilon_{4} X & \varepsilon_{4} X M & \eta H_{2}^{T} & 0
\end{array}\right], \\
& \Psi_{22}=\operatorname{diag}\left(-e^{\alpha \tau_{2}} \bar{Q},-I,-\eta I,-\eta I,-\rho^{2} I\right), \\
& \Pi_{11}=R+Q+\bar{Q}-\alpha P+\varepsilon_{1} X A+\varepsilon_{1} A^{T} X^{T}, \\
& \Pi_{12}=P-\varepsilon_{1} X+\varepsilon_{2} A^{T} X^{T}, \\
& \Pi_{14}=\varepsilon_{1} X B+\varepsilon_{4} A^{T} X^{T}, \\
& \Pi_{22}=-\varepsilon_{2} X-\varepsilon_{2} X^{T} \text {, } \\
& \Pi_{24}=\varepsilon_{2} X B-\varepsilon_{4} X^{T} \text {, } \\
& \Pi_{44}=(\mu-1) e^{a \tau_{2}} R+\varepsilon_{4} X B+\varepsilon_{4} B^{T} X^{T} .
\end{aligned}
$$

Proof. Let us define the Lyapunov functional candidate as following:

$$
\begin{aligned}
V(t)= & x^{T}(t) P x(t)+\int_{t-\tau(t)}^{t} e^{\alpha(t-s)} x^{T}(s) R x(s) d s \\
& +\int_{t-\tau_{1}}^{t} e^{\alpha(t-s)} x^{T}(s) Q x(t) d s \\
& +\int_{t-\tau_{2}}^{t} e^{\alpha(t-s)} x^{T}(s) \bar{Q} x(t) d s .
\end{aligned}
$$

Then

$$
\begin{aligned}
J= & \dot{V}(t)-\alpha V(t) \\
= & 2 x^{T}(t) P \dot{x}(t)+x^{T}(t)(R+Q+\bar{Q}-\alpha P) x(t) \\
& -(1-\dot{\tau}(t)) e^{\alpha \tau(t)} x^{T}(t-\tau(t)) R x(t-\tau(t)) \\
& -e^{\alpha \tau_{1}} x^{T}\left(t-\tau_{1}\right) Q x\left(t-\tau_{1}\right) \\
& -e^{\alpha \tau_{2}} x^{T}\left(t-\tau_{2}\right) \bar{Q} x\left(t-\tau_{2}\right) \\
\leq & 2 x^{T}(t) P \dot{x}(t)+x^{T}(t)(R+Q+\bar{Q}-\alpha P) x(t) \\
& -(1-\mu) e^{\alpha \tau_{2}} x^{T}(t-\tau(t)) R x(t-\tau(t)) \\
& -e^{\alpha \tau_{2}} x^{T}\left(t-\tau_{2}\right) \bar{Q} x\left(t-\tau_{2}\right) \\
& -e^{\alpha \tau_{1}} x^{T}\left(t-\tau_{1}\right) Q x\left(t-\tau_{1}\right) .
\end{aligned}
$$

Moreover, from (2), we have

$$
\rho^{2} x^{T}(t) x(t)-f^{T}(x(t)) f(x(t)) \geq 0 .
$$

Combining (11) and (12), we get

$$
J \leq \xi^{T}(t) \Pi_{1} \xi(t),
$$

where

$$
\begin{aligned}
& \xi^{T}(t)=\left[\xi_{1}^{T}(t), \xi_{2}^{T}(t)\right], \\
& \xi_{1}^{T}(t)=\left[x^{T}(t), \dot{x}^{T}(t)\right], \\
& \xi_{2}^{T}(t)=\left[x^{T}\left(t-\tau_{1}\right), x^{T}(t-\tau(t)), x^{T}\left(t-\tau_{2}\right), f^{T}(x(t))\right], \\
& \Pi_{1}=\left[\begin{array}{cccccc}
J_{11} & P & 0 & 0 & 0 & 0 \\
* & 0 & 0 & 0 & 0 & 0 \\
* & * & -e^{\alpha \tau_{1}} Q & 0 & 0 & 0 \\
* & * & * & J_{44} & 0 & 0 \\
* & * & * & * & -e^{\alpha \tau_{2}} \bar{Q} & 0 \\
* & * & * & * & * & -I
\end{array}\right], \\
& J_{11}=R-\alpha P+\rho^{2} I+Q+\bar{Q}, \\
& J_{44}=(\mu-1) e^{\alpha \tau_{2}} R \text {. }
\end{aligned}
$$

On the other hand, from (7), we obtain

$$
\left[\begin{array}{llllll}
\bar{A} & -I & 0 & \bar{B} & 0 & I
\end{array}\right] \xi(t)=0 .
$$

According to Lemma 1, if there exist parameters $\varepsilon_{1}$, $\varepsilon_{2}, \varepsilon_{3}, \varepsilon_{4}$, and the symmetric positive definite matrices $X$ satisfying the following inequality, then 
$\xi^{T}(t) \Pi_{1} \xi(t)$ will be negative at the points satisfying

(14)

$$
\bar{\Pi}_{1}=\Pi_{1}+\left[\begin{array}{c}
\varepsilon_{1} X \\
\varepsilon_{2} X \\
\varepsilon_{3} X \\
\varepsilon_{4} X \\
0 \\
0
\end{array}\right]\left[\begin{array}{llllll}
\bar{A} & -I & 0 & \bar{B} & 0 & I
\end{array}\right]+\left[\begin{array}{c}
\bar{A}^{T} \\
-I \\
0 \\
\bar{B}^{T} \\
0 \\
I
\end{array}\right]
$$$$
\cdot\left[\varepsilon_{1} X^{T} \quad \varepsilon_{2} X^{T} \quad \varepsilon_{3} X^{T} \quad \varepsilon_{4} X^{T} \quad 0 \quad 0\right]<0,
$$

which is equivalent to

$$
\bar{\Pi}_{1}=\left[\begin{array}{cccccc}
\bar{\Pi}_{11} & \bar{\Pi}_{12} & \bar{\Pi}_{13} & \bar{\Pi}_{14} & 0 & \varepsilon_{1} X \\
* & \bar{\Pi}_{22} & \bar{\Pi}_{23} & \bar{\Pi}_{24} & 0 & \varepsilon_{2} X \\
* & * & \bar{\Pi}_{33} & \bar{\Pi}_{34} & 0 & \varepsilon_{3} X \\
* & * & * & \bar{\Pi}_{44} & 0 & \varepsilon_{4} X \\
* & * & * & * & \bar{\Pi}_{55} & 0 \\
* & * & * & * & * & -I
\end{array}\right]<0
$$

where

$\bar{\Pi}_{11}=R-\alpha P+\rho^{2} I+Q+\bar{Q}+\varepsilon_{1} X \bar{A}+\varepsilon_{1} \bar{A}^{T} X^{T}$,

$\bar{\Pi}_{12}=P-\varepsilon_{1} X+\varepsilon_{1} \bar{A}^{T} X^{T}$,

$\bar{\Pi}_{13}=\varepsilon_{1} \bar{A}^{T} X^{T}$,

$\bar{\Pi}_{14}=\varepsilon_{1} X \bar{B}+\varepsilon_{4} \bar{A}^{T} X^{T}$,

$\bar{\Pi}_{22}=-\varepsilon_{2} X-\varepsilon_{2} X^{T}$,

$\bar{\Pi}_{23}=-\varepsilon_{3} X^{T}$,

$\bar{\Pi}_{24}=\varepsilon_{2} X \bar{B}-\varepsilon_{3} X^{T}$,

$\bar{\Pi}_{33}=-e^{\alpha \tau_{1}} Q$,

$\bar{\Pi}_{34}=\varepsilon_{3} X \bar{B}$,

$\bar{\Pi}_{44}=(\mu-1) e^{\alpha \tau_{2}} R+\varepsilon_{4} X \bar{B}+\varepsilon_{4} \bar{B}^{T} X^{T}$,

$\bar{\Pi}_{55}=-e^{\alpha \tau_{2}} \bar{Q}$.

The above inequality can be rewritten as follows:

$$
\bar{\Pi}_{1}=\Gamma+\Theta_{1} S(t) \Theta_{2}+\Theta_{2}^{T} S^{T}(t) \Theta_{1}^{T},
$$

where

$$
\begin{aligned}
& \Theta_{1}^{T}=\left[\varepsilon_{1} M^{T} X^{T}, \varepsilon_{2} M^{T} X^{T}, \varepsilon_{3} M^{T} X^{T}, \varepsilon_{4} M^{T} X^{T}, 0,0\right], \\
& \Theta_{2}=\left[\begin{array}{llllll}
H_{1} & 0 & 0 & H_{2} & 0 & 0
\end{array}\right],
\end{aligned}
$$

$$
\Gamma=\left[\begin{array}{cccccc}
\hat{\Pi}_{11} & \hat{\Pi}_{12} & \varepsilon_{3} A^{T} X^{T} & \hat{\Pi}_{14} & 0 & \varepsilon_{1} X \\
* & \bar{\Pi}_{22} & -\varepsilon_{3} X^{T} & \hat{\Pi}_{24} & 0 & \varepsilon_{2} X \\
* & * & \bar{\Pi}_{33} & \varepsilon_{3} X B & 0 & \varepsilon_{3} X \\
* & * & * & \hat{\Pi}_{44} & 0 & \varepsilon_{4} X \\
* & * & * & * & \bar{\Pi}_{55} & 0 \\
* & * & * & * & * & -I
\end{array}\right],
$$

$$
\begin{aligned}
& \hat{\Pi}_{11}=R-\alpha P+\rho^{2} I+Q+\bar{Q}+\varepsilon_{1} X A+\varepsilon_{1} A^{T} X^{T}, \\
& \hat{\Pi}_{12}=P-\varepsilon_{1} X+\varepsilon_{1} A^{T} X^{T}, \\
& \hat{\Pi}_{14}=\varepsilon_{1} X B+\varepsilon_{4} A^{T} X^{T}, \\
& \hat{\Pi}_{24}=\varepsilon_{2} X B-\varepsilon_{3} X^{T}, \\
& \hat{\Pi}_{44}=(\mu-1) e^{\alpha \tau_{2}} R+\varepsilon_{4} X B+\varepsilon_{4} B^{T} X^{T} .
\end{aligned}
$$

According to Lemma 2, we can get

$$
\bar{\Pi}_{1} \leq \Gamma+\eta^{-1} \Theta_{1} \Theta_{1}^{T}+\eta \Theta_{2}^{T} \Theta_{2} .
$$

Using Schur complement lemma, from (8) we have $\Pi_{1}<0$. Hence, it follows that

$$
\dot{V}(x(t))-\alpha V(x(t)) \leq 0,
$$

which yields

$$
V(t) \leq e^{\alpha t} V(0) .
$$

From (10), we have

$$
\begin{aligned}
V(0)= & x^{T}(0) P x(0)+\int_{-\tau(0)}^{0} e^{-\alpha s} x^{T}(s) R x(s) d s \\
& +\int_{-\tau_{1}}^{0} e^{-\alpha s} x^{T}(s) Q x(s) d s \\
& +\int_{-\tau_{2}}^{0} e^{-\alpha s} x^{T}(s) \bar{Q} x(s) d s \\
\leq & x^{T}(0) P x(0)+\int_{-\tau_{2}}^{0} e^{-\alpha s} x^{T}(s) R x(s) d s \\
& +\int_{-\tau_{1}}^{0} e^{-\alpha s} x^{T}(s) Q x(s) d s \\
& +\int_{-\tau_{2}}^{0} e^{-\alpha s} x^{T}(s) \bar{Q} x(s) d s \\
\leq & \left(\lambda_{2}+\lambda_{3} \tau_{2} e^{-\alpha \tau_{2}}+\lambda_{4} \tau_{1} e^{-\alpha \tau_{1}}+\lambda_{5} \tau_{2} e^{-\alpha \tau_{2}}\right)\|\phi\|^{2} \\
= & \lambda\|\phi\|^{2},
\end{aligned}
$$

where

$$
\begin{aligned}
& \lambda=\lambda_{2}+\lambda_{3} \tau_{2} e^{-\alpha \tau_{2}}+\lambda_{4} \tau_{1} e^{-\alpha \tau_{1}}+\lambda_{5} \tau_{2} e^{-\alpha \tau_{2}}, \\
& \lambda_{2}=\lambda_{\text {max }}(P), \quad \lambda_{3}=\lambda_{\text {max }}(R), \\
& \lambda_{4}=\lambda_{\text {max }}(Q), \quad \lambda_{5}=\lambda_{\text {max }}(\bar{Q}) .
\end{aligned}
$$


Combine (19) and (20), we have

$$
V(t) \leq \lambda e^{\alpha t}\|\phi\|^{2} .
$$

On the other hand

$$
V(t) \geq \lambda_{1}\|x(t)\|^{2},
$$

where $\lambda_{1}=\lambda_{\min }(P)$.

From (19) and (20), we have

$$
\lambda_{1}\|x(t)\|^{2} \leq \lambda e^{\alpha t}\|\phi\|^{2} .
$$

The above inequality can be rewritten as follows:

$$
\|x(t)\| \leq \sqrt{\frac{\lambda}{\lambda_{1}}} e^{\frac{\alpha}{2}}\|\phi\| .
$$

By Definition 1 and inequality (23), we have that system (7) is exponentially stable with decay rate $\kappa=-\frac{\alpha}{2}$. This completes the proof.

Remark 1. When $\tau(t)=\tau$, the system (7) will be modified as follows:

$$
\left\{\begin{array}{l}
\dot{x}(t)=\bar{A} x(t)+\bar{B} x(t-\tau)+f(x(t)), \\
x(t)=\phi(t), t \in[-\tau, 0] .
\end{array}\right.
$$

The following corollary can be obtained immediately.

Corollary 1. For given parameters $\varepsilon_{1}, \varepsilon_{2}, \varepsilon_{3}, \rho$, $\alpha<0, \tau>0$, and $u<1$, the system (24) is exponentially stable with decay rate $\kappa=-\frac{\alpha}{2}$, if there exist symmetric positive definite matrices $P$, $R, X$ and a positive scalars $\eta$, such that the following LMI holds:

$\Omega=\left[\begin{array}{cccccc}\Omega_{11} & \Omega_{12} & \Omega_{13} & \varepsilon_{1} X & \varepsilon_{1} X M & \eta H_{1}^{T} \\ * & \Omega_{22} & \Omega_{23} & \varepsilon_{2} X & \varepsilon_{2} X M & 0 \\ * & * & \Omega_{33} & \varepsilon_{3} X & \varepsilon_{3} X M & \eta H_{2}^{T} \\ * & * & * & -I & 0 & 0 \\ * & * & * & * & -\eta I & 0 \\ * & * & * & * & * & -\eta I\end{array}\right]<0$,

where

$$
\begin{aligned}
& \Omega_{11}=R-\alpha P+\rho^{2} I+\varepsilon_{1} X A+\varepsilon_{1} A^{T} X^{T}, \\
& \Omega_{12}=P-\varepsilon_{1} X+\varepsilon_{2} A^{T} X^{T}, \\
& \Omega_{13}=\varepsilon_{1} X B+\varepsilon_{3} A^{T} X^{T}, \quad \Omega_{22}=-\varepsilon_{2} X-\varepsilon_{2} X^{T}, \\
& \Omega_{23}=\varepsilon_{2} X B-\varepsilon_{3} X^{T}, \quad \Omega_{23}=\varepsilon_{2} X B-\varepsilon_{3} X^{T} .
\end{aligned}
$$

Proof. The proof follows from the similar lines of theorem 1 by picking

$$
V(t)=x^{T}(t) P x(t)+\int_{t-\tau}^{t} e^{\alpha(t-s)} x^{T}(s) R x(s) d s .
$$

The proof is similar that of Theorem 1, and the detailed proof is omitted.

We consider a state feedback controller as following

$$
u(t)=K x(t) .
$$

Substituting (25) into the system (1) leads to the following closed-loop system

$$
\left\{\begin{array}{l}
\dot{x}(t)=[\tilde{A}+\Delta A] x(t)+\bar{B} x(t-\tau(t))+f(x(t)), \\
x(t)=\phi(t), \quad t \in\left[-\tau_{2}, 0\right] .
\end{array}\right.
$$

where $\tilde{A}=A+H K$.

For the system (26), we can obtain following theorem.

Theorem 2. For given parameters $\varepsilon_{1}, \varepsilon_{2}, \varepsilon_{3}, \varepsilon_{4}$, $\rho>0, \alpha<0,0<\tau_{1} \leq \tau_{2}$, and $\mu<1$, the closed-loop system (26) is exponentially stable with decay rate $\kappa=-\frac{\alpha}{2}$, if there exist symmetric matrices $\tilde{P}>0$, $\tilde{\bar{Q}}>0, \tilde{Q}>0, \quad \tilde{R}>0, \quad \tilde{X}>0$, any matrix $Y$ and a positive scalar $\tilde{\eta}$, such that the following LMI holds:

$\Xi=\left[\begin{array}{ccccc}\Xi_{11} & \Xi_{12} & \Xi_{13} & \Xi_{14} & 0 \\ * & -2 \varepsilon_{2} \tilde{X} & \varepsilon_{3} \tilde{X} & \Xi_{24} & 0 \\ * & * & -e^{\alpha \tau_{1}} Q & \varepsilon_{3} B \tilde{X} & 0 \\ * & * & * & \Xi_{44} & 0 \\ * & * & * & * & -e^{\alpha \tau_{2}} \tilde{\bar{Q}} \\ * & * & * & * & * \\ * & * & * & * & * \\ * & * & * & * & * \\ * & * & * & * & *\end{array}\right.$

$$
\left.\begin{array}{cccc}
\varepsilon_{1} I & \varepsilon_{1} \tilde{\eta} M & \tilde{X} H_{1}^{T} & \tilde{X} \\
\varepsilon_{2} I & \varepsilon_{2} \tilde{\eta} M & 0 & 0 \\
\varepsilon_{3} I & \varepsilon_{3} \tilde{\eta} M & 0 & 0 \\
\varepsilon_{4} I & \varepsilon_{4} \tilde{\eta} M & \tilde{X} H_{2}^{T} & 0 \\
0 & 0 & 0 & 0 \\
-I & 0 & 0 & 0 \\
* & -\tilde{\eta} I & 0 & 0 \\
* & * & -\tilde{\eta} I & 0 \\
* & * & * & -\rho^{2} I
\end{array}\right]<0,
$$


where

$$
\begin{aligned}
\Xi_{11}= & \tilde{R}-\alpha \tilde{P}+\tilde{Q}+\tilde{\bar{Q}}+\varepsilon_{1} A \tilde{X}+\varepsilon_{1} H Y \\
& +\varepsilon_{1} \tilde{X} A^{T}+\varepsilon_{1} Y^{T} H^{T}, \\
\Xi_{12}= & \tilde{P}-\varepsilon_{1} \tilde{X}+\varepsilon_{2} \tilde{X} A^{T}+\varepsilon_{2} Y^{T} H^{T}, \\
\Xi_{13}= & \varepsilon_{3} \tilde{X} A^{T}+\varepsilon_{3} Y^{T} H^{T}, \\
\Xi_{14}= & \varepsilon_{1} B \tilde{X}+\varepsilon_{4} \tilde{X} A^{T}+\varepsilon_{4} Y^{T} H^{T}, \\
\Xi_{24}= & \varepsilon_{2} B \tilde{X}-\varepsilon_{4} \tilde{X}, \\
\Xi_{44}= & (\mu-1) e^{\alpha \tau_{2}} \tilde{R}+\varepsilon_{4} B \tilde{X}+\varepsilon_{4} \tilde{X} B^{T} .
\end{aligned}
$$

Moreover, the controller gain matrix is designed by

$$
K=Y \tilde{X}^{-1} .
$$

Proof. By replacing $\tilde{A}$ instead of $A$ in the inequality (8), we can obtaint

$$
\tilde{\Pi}=\left[\begin{array}{ccccc}
\tilde{\Pi}_{11} & \tilde{\Pi}_{12} & \tilde{\Pi}_{13} & \tilde{\Pi}_{14} & 0 \\
* & \Pi_{22} & \varepsilon_{3} X^{T} & \Pi_{24} & 0 \\
* & * & -e^{\alpha \tau_{1}} Q & \varepsilon_{3} X B & 0 \\
* & * & * & \Pi_{44} & 0 \\
* & * & * & * & -e^{\alpha \tau_{2}} \bar{Q} \\
* & * & * & * & * \\
* & * & * & * & * \\
* & * & * & * & * \\
* & * & * & * & * \\
& \varepsilon_{1} X & \varepsilon_{1} X M & \eta H_{1}^{T} & I \\
& \varepsilon_{2} X & \varepsilon_{2} X M & 0 & 0 \\
\varepsilon_{3} X & \varepsilon_{3} X M & 0 & 0 \\
\varepsilon_{4} X & \varepsilon_{4} X M & \eta H_{2}^{T} & 0 \\
0 & 0 & 0 & 0 \\
-I & 0 & 0 & 0 \\
* & -\eta I & 0 & 0 \\
* & * & -\eta I & 0 \\
* & * & * & -\rho^{2} I
\end{array}\right]<0,
$$

where

$$
\begin{aligned}
& \tilde{\Pi}_{11}=R-\alpha P+Q+\bar{Q}+\varepsilon_{1} X \tilde{A}+\varepsilon_{1} \tilde{A}^{T} X^{T}, \\
& \tilde{\Pi}_{12}=P-\varepsilon_{1} X+\varepsilon_{2} \tilde{A}^{T} X^{T}, \tilde{\Pi}_{13}=\varepsilon_{3} \tilde{A}^{T} X^{T} .
\end{aligned}
$$

Denote $\tilde{\eta}=\eta^{-1}, \tilde{R}=X^{-1} R X^{-1}, \tilde{Q}=X^{-1} Q X^{-1}$,

$$
\tilde{\bar{Q}}=X^{-1} \bar{Q} X^{-1}, \tilde{P}=X^{-1} P X^{-1}, \tilde{X}=X^{-1} \text {. }
$$

Now Pre- and post-multiplying (29) by

$$
\operatorname{diag}\left(X^{-1}, X^{-1}, X^{-1}, X^{-1}, X^{-1}, I, \eta^{-1}, \eta^{-1}, I\right),
$$

we get that

$\hat{\Pi}=\left[\begin{array}{ccccc}\hat{\Pi}_{11} & \hat{\Pi}_{12} & \hat{\Pi}_{13} & \hat{\Pi}_{14} & 0 \\ * & \hat{\Pi}_{22} & -\varepsilon_{3} \tilde{X}^{T} & \hat{\Pi}_{24} & 0 \\ * & * & -e^{\alpha \tau_{1}} \tilde{Q} & \varepsilon_{3} B \tilde{X} & 0 \\ * & * & * & \hat{\Pi}_{44} & 0 \\ * & * & * & * & -e^{\alpha \tau_{2}} \tilde{Q} \\ * & * & * & * & * \\ * & * & * & * & * \\ * & * & * & * & * \\ * & * & * & * & *\end{array}\right.$

$$
\left.\begin{array}{cccc}
\varepsilon_{1} I & \varepsilon_{1} \tilde{\eta} M & \tilde{X} H_{1}^{T} & \tilde{X} \\
\varepsilon_{2} I & \varepsilon_{2} \tilde{\eta} M & 0 & 0 \\
\varepsilon_{3} I & \varepsilon_{3} \tilde{\eta} M & 0 & 0 \\
\varepsilon_{4} I & \varepsilon_{4} \tilde{\eta} M & \tilde{X} H_{2}^{T} & 0 \\
0 & 0 & 0 & 0 \\
-I & 0 & 0 & 0 \\
* & -\tilde{\eta} I & 0 & 0 \\
* & * & -\tilde{\eta} I & 0 \\
* & * & * & -\rho^{2} I
\end{array}\right]<0,
$$

where

$$
\begin{aligned}
\hat{\Pi}_{11}= & \tilde{R}-\alpha \tilde{P}+\tilde{Q}+\tilde{\bar{Q}}+\varepsilon_{1} A \tilde{X}+\varepsilon_{1} H K \tilde{X}+\varepsilon_{1} \tilde{X} A^{T} \\
& +\varepsilon_{1} \tilde{X} K^{T} H^{T}, \\
\hat{\Pi}_{12}= & \tilde{P}-\varepsilon_{1} \tilde{X}+\varepsilon_{2} \tilde{X} A^{T}+\varepsilon_{2} \tilde{X} K^{T} H^{T}, \\
\hat{\Pi}_{13}= & \varepsilon_{3} \tilde{X} A^{T}+\varepsilon_{3} \tilde{X} K^{T} H^{T}, \\
\hat{\Pi}_{14}= & \varepsilon_{1} B \tilde{X}+\varepsilon_{4} \tilde{X} A^{T}+\varepsilon_{4} \tilde{X} K^{T} H^{T}, \\
\hat{\Pi}_{22}= & -\varepsilon_{2} \tilde{X}-\varepsilon_{2} \tilde{X}{ }^{T}, \\
\hat{\Pi}_{24}= & \varepsilon_{2} B \tilde{X}-\varepsilon_{4} \tilde{X}, \\
\hat{\Pi}_{44}= & (\mu-1) e^{\alpha \tau_{2}} \tilde{R}+\varepsilon_{4} B \tilde{X}+\varepsilon_{4} \tilde{X} B^{T} .
\end{aligned}
$$

Let $K \tilde{X}=Y$. We obtain (27). This completes the proof.

\section{Numerical example}

In this part, we will use a numerical example to verify the practicability of the forward variation theorem.

Consider the system (26) with the following parameters

$$
A=\left[\begin{array}{cc}
5 & 11 \\
9 & -2
\end{array}\right], B=\left[\begin{array}{cc}
1 & 4 \\
-3 & -5
\end{array}\right], \quad H=\left[\begin{array}{cc}
2 & 4 \\
-2 & 3
\end{array}\right],
$$




$$
\begin{aligned}
& M=\left[\begin{array}{cc}
1 & -9 \\
1 & 3
\end{array}\right], \quad f(x(t))=\left[\begin{array}{c}
0.12 \sin \left(x_{1}(t)\right) \\
0.1 \sin \left(x_{2}(t)\right)
\end{array}\right], \\
& H_{1}=\left[\begin{array}{cc}
0.6 & 0 \\
0 & 0.6
\end{array}\right], \quad H_{2}=\left[\begin{array}{cc}
0.2 & 0 \\
0 & 0.2
\end{array}\right], \\
& S(t)=\left[\begin{array}{ll}
1 & 0 \\
0 & 1
\end{array}\right], \quad \tau(t)=0.11-0.1 \sin (t) .
\end{aligned}
$$

Consider the decay rate $\kappa=0.02$. Let us take $\mu=0.4, \varepsilon_{1}=0.9, \varepsilon_{2}=\varepsilon_{3}=0.1, \varepsilon_{4}=0.2, \rho=0.5$.

Then by solving the LMI (27) with the use of MATLAB LMI toolbox, we can obtain the following feasible solution

$$
\begin{aligned}
\tilde{P} & =\left[\begin{array}{ll}
0.2713 & 0.0403 \\
0.0403 & 0.6259
\end{array}\right], \\
\tilde{Q} & =\left[\begin{array}{cc}
1.1233 & -0.1300 \\
-0.1300 & 0.5341
\end{array}\right], \\
\tilde{\bar{Q}} & =\left[\begin{array}{ll}
0.2779 & 0.0206 \\
0.0206 & 0.1096
\end{array}\right], \\
\tilde{R} & =\left[\begin{array}{ll}
0.8233 & 0.2314 \\
0.2314 & 2.0154
\end{array}\right], \\
\tilde{X} & =\left[\begin{array}{cc}
0.1854 & -0.2060 \\
-0.2060 & 0.3163
\end{array}\right], \\
Y & =\left[\begin{array}{ll}
-1.2161 & -0.4915 \\
-0.4915 & -0.8029
\end{array}\right], \\
\eta & =0.9301 .
\end{aligned}
$$

The control gain is

$$
K=\left[\begin{array}{ll}
-30.0219 & -21.1118 \\
-19.8259 & -15.4542
\end{array}\right] \text {. }
$$

According Theorem 2, the closed-loop system (26) is exponentially stable.

Fig. 1 shows the simulation results of the state trajectory of the system (26) under the control gain proposed above. From Fig. 1, it is evident that the state responses converge quickly to the equilibrium point.

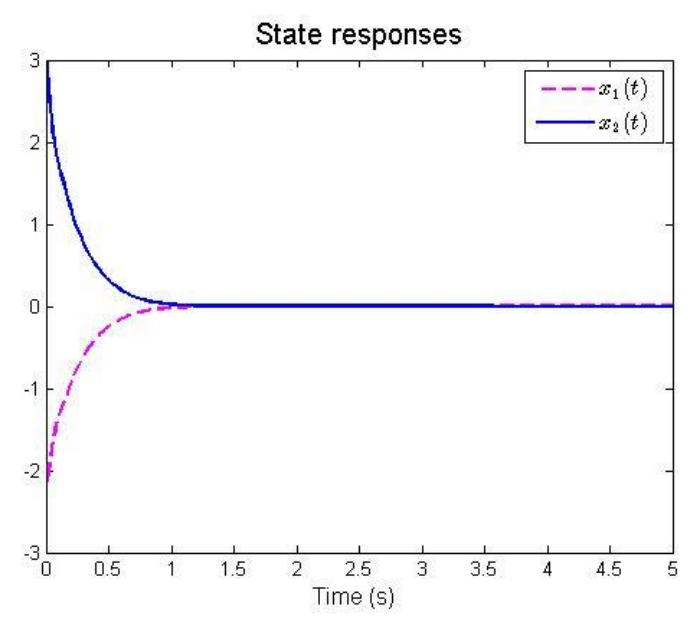

Fig.1. The state trajectory of system (26)

\section{Conclusion}

This paper deals with the problem of the exponential stabilization for a class of nonlinear uncertain systems with time-varying delay. In this paper, we apply the Finsler's lemma to deal with the system's uncertainty. We proposed a new criterion for the exponential stability of nonlinear uncertain system, and the sufficient condition of exponential stability for a class of nonlinear uncertain systems with constant time delay is given. Then, sufficient conditions of robust exponential stabilization for nonlinear uncertain systems with time-varying delay are derived. The state feedback control gain is designed. Finally, a numerical example is given to illustrate the effectiveness of the method.

\section{Acknowledgments}

This work was supported by the Natural Science Foundation of Tianjin under Grant No. 18JCYBJC88000.

\section{References}

[1] X. Liu, K. Zhang, Stabilization of nonlinear timedelay systems: distributed-delay dependent impulsive control, Syst. Control Lett, Vol. 120, 2018, pp. 17-22.

[2] R. Yang, L. Sun, Finite-time robust control of a class of nonlinear time-delay systems via 
Lyapunov functional method, J. Frankl, Inst. Vol. 356, 2018, pp. 1155-117.

[3] W. He, J. Guo, Z. Xiang, Global sampled-data output feedback stabilization for a class of stochastic nonlinear systems with time-varying delay, Journal of the Franklin Institute, Vol. 356, 2019, pp. 292-308.

[4] F. Gao, Y. Wu, Further results on global state feedback stabilization of high-order nonlinear systems with time-varying delays, ISA Transactions, Vol. 55, 2015, pp. 41-48.

[5] Y. Dong, T. Li, S. Mei, Exponential stabilization and $L_{2}$-gain for uncertain switched nonlinear systems with interval time-varying delay, Mathematical Methods in the Applied Sciences, Vol. 39, 2016, pp. 3836-3854.

[6] Y. Tian, Y. Cai, Y. Sun, Stability of switched nonlinear time-delay systems with stable and unstable subsystems, Nonlinear Analysis: Hybrid Systems, Vol. 24, 2017, pp. 58-68.

[7] Y. Dong, L. Guo, J. Hao, Robust exponential stabilization for uncertain neutral neural networks with time-varying delays by periodically intermittent control, Neural Computing and Application, Vol. 32, 2020, pp. 2651-2664.

[8] R. Sanz, P. García and M. Krstic, Observation and stabilization of LTV systems with timevarying measurement delay, Automatica, Vol. 103, 2019, pp. 573-579.

[9] O. M. Kwon, M.J. Park, Ju H. Park, S.M. Lee, E.J. Cha, Improved approaches to stability criteria for neural networks with time-varying delays. Journal of the Franklin Institute, Vol. 350, 2013, pp. 2710-2735.

[10] H. Gholami and M. H. Shafiei, Finite-time $H_{\infty}$ static and dynamic output feedback control for a class of switched nonlinear time-delay systems, Applied Mathematics and Computation, Vol. 389, 2021, pp. 125557.

[11] Y. Huang, S. Fu, Y. Shen, Finite-time $H_{\infty}$ control for one-sided Lipschitz systems with auxiliary matrices, Neurocomputing, Vol. 194, 2016, pp. 207-217.
[12] L. Hou, G. Zong, Y. Wu, Robust exponential stability analysis of discrete-time switched Hopfield neural networks with time delay. Nonlinear Anal Hybrid Systems, Vol. 5, 2011, pp. 525-534

[13] J. Zhang, P. Shi, J. Qiu, S.K. Nguang, A novel observer-based output feedback controller design for discrete-time fuzzy systems, IEEE Trans. Fuzzy Syst, Vol. 23, No. 1, 2015, pp. 223-229.

[14] T. Botmart, P. Niamsup, V.N. Phat, Delaydependent exponential stabilization for uncertain linear systems with interval nondifferentiable time-varying delays, Applied Mathematics and Computation, Vol. 217, 2011, pp. 8236-8247.

[15] Y. Dong, S. Liang, L. Guo, W. Wang, Exponential stability and stabilization for uncertain discrete-time periodic systems with time-varying delay. IMA J Math Control Inf. Vol. 35, 2018, pp. 963-986.

[16] L. V. Hien, V. N. Phat, Exponential stability and stabilization of a class of uncertain linear timedelay systems, J. Frankl. Inst, Vol. 346, No. 6, 2009, pp. 611-625.

[17] S. Mondie, V. L. Kharitonov, Exponential estimates for retarded time-delay systems: an LMI approach, IEEE Trans. Autom. Control, Vol. 50, No. 2, 2005, pp. 268-273.

[18] Y. Huang, S. Fu, Y. Shen, Finite-time $H_{\infty}$ control for one-sided Lipschitz systems with auxiliary matrices, Neurocomputing, Vol. 194, 2016, pp. 207-217.

\section{Creative Commons Attribution License 4.0 (Attribution 4.0 International, CC BY 4.0)}

This article is published under the terms of the Creative Commons Attribution License 4.0 https://creativecommons.org/licenses/by/4.0/deed.en US 